ARTICLE

\title{
One-way dependent clusters and stability of cluster synchronization in directed networks
}

\author{
Matteo Lodi (i) ${ }^{1}$, Francesco Sorrentino (i) ${ }^{2} \&$ Marco Storace (iD ${ }^{1 凶}$
}

Cluster synchronization in networks of coupled oscillators is the subject of broad interest from the scientific community, with applications ranging from neural to social and animal networks and technological systems. Most of these networks are directed, with flows of information or energy that propagate unidirectionally from given nodes to other nodes. Nevertheless, most of the work on cluster synchronization has focused on undirected networks. Here we characterize cluster synchronization in general directed networks. Our first observation is that, in directed networks, a cluster $A$ of nodes might be one-way dependent on another cluster $B$ : in this case, A may remain synchronized provided that B is stable, but the opposite does not hold. The main contribution of this paper is a method to transform the cluster stability problem in an irreducible form. In this way, we decompose the original problem into subproblems of the lowest dimension, which allows us to immediately detect inter-dependencies among clusters. We apply our analysis to two examples of interest, a human network of violin players executing a musical piece for which directed interactions may be either activated or deactivated by the musicians, and a multilayer neural network with directed layer-to-layer connections.

\footnotetext{
${ }^{1}$ DITEN, University of Genoa, Genova, Italy. ${ }^{2}$ Mechanical Engineering Department, University of New Mexico, Albuquerque, NM, USA. ${ }^{凶}$ email: marco.storace@unige.it
} 
S ynchronization is a pervasive phenomenon in networks of natural and man-made dynamical systems ${ }^{1}$, which often enables complex functions corresponding to patterns of coordinated activity. Some of these systems require complete (or full) synchronization among all network components to properly function: examples include the coherent firing of neural populations ${ }^{2}$, synchronous behaviors in networks of chemical oscillators $^{3}$, the concerted rhythmical flashing on and off of firefly swarms $^{4}$, or frequency-locked power generation ${ }^{5}$. In other cases, such as higher brain centers of the nervous system ${ }^{6}$, rhythmic activity and synchronization may correspond to a pathological condition. Other systems rely on cluster (or partial) synchronization (CS), where different groups exhibit different, yet synchronized, internal behaviors. For instance, a large corpus of studies has shown the connection of neural synchronization of brain areas with cognition ${ }^{6}$, animal locomotion is controlled to some extent by central pattern generators that impose a given gait by exploiting synchronous clusters of neurons ${ }^{7-9}$, and connected vehicle systems often give rise to $\mathrm{CS}^{10}$. The study of $\mathrm{CS}$ is therefore relevant to analyze and control natural (ecological, immune, neural, and cellular) ${ }^{11}$ and artificial ${ }^{12-14}$ systems.

A network of dynamical systems can be modeled through (i) a graph (where an element is called a node and a connection is called an edge or link, or an arrow if oriented), which is an abstract description of its architecture or topology, and (ii) a set of dynamical equations describing the time evolution of each node and, possibly, also of each connection. The interplay between the network topology and the dynamics of the nodes and of their interactions determines the complex dynamics emergent in all the systems described above.

A large body of literature has investigated stability of the cluster-synchronous solution for undirected networks ${ }^{15-24}$, corresponding to graphs whose edges indicate a two-way relationship. However, most real networks are directed and are described by graphs (also called digraphs) whose edges have a direction that indicates a one-way relationship. In many fields, from systems biology to the engineering of distributed technology 25,26 , the corresponding physical systems do not always exhibit a bidirectional flow of information/energy, thus leading to preferred paths for routing of information/energy. For example, neural, gene regulatory, metabolic, and epidemic networks are all directed. Understanding the conditions for the emergence of stable synchronized clusters in these networks is of considerable importance.

Following ref. ${ }^{18}$, a fundamental issue is to be able to reduce the dimensionality of the stability analysis for synchronized clusters. However, it remains an open problem how such reduction can be obtained for directed networks. Here we bridge this gap by decoupling the CS stability problem into lower-dimensional problems. References ${ }^{18,21,22}$ study undirected networks and explain how to analyze the stability of any CS solution by introducing a transformation-based on the irreducible representation (IRR) of the network symmetry group-from the node coordinate system to the IRR coordinate system.

The methods based on IRR transformations ${ }^{17,18,23}$ have been applied to undirected networks. For these networks, it is also possible to understand if two clusters $C_{1}$ and $C_{2}$ are intertwined, i.e., if desynchronization of cluster $C_{1}$ leads to loss of synchrony in cluster $C_{2}$ and vice versa ${ }^{18,27}$.

The main contribution of this paper is a method for analyzing the stability of synchronized clusters in directed multilayer networks with general structure. This method is based on two cornerstones. The first one is a generalization of the concept of intertwined clusters, which takes into account directionality: we define one-way dependent clusters, meaning that the breaking (i.e., loss of stability) of a synchronized cluster $C_{1}$ causes the loss of synchrony of another cluster $C_{2}$, but not vice versa. The second cornerstone is a method to build up the matrices that we use to analyze cluster stability in directed networks through linearization: owing to this method, we solve a problem still open, namely how to find a coordinate transformation that separates as much as possible perturbation modes in the stability analysis. This method also allows easy detection (by inspection of certain matrices) of interdependencies between clusters in directed networks, and so a classification of pairs of clusters as independent or intertwined or one-way dependent in terms of their stability. This method enables the analysis of a class of systems, for which, to the best of our knowledge, other analysis tools are not available, thus bridging a gap toward the analysis of biological, social, and technological systems described by directed networks. Alternative changes of variables ${ }^{27}$ are simpler to compute but do not ensure that the coordinate transformation is optimal in the sense specified above.

We apply the proposed method to two illustrative case studies, namely two networks from different fields. The first one is a single-layer network with delays and delay-dependent connections modeling a group of violin players ${ }^{28}$. The second one is a two-layer network of neurons that can also produce chimera states $^{29,30}$. In both cases, our method provides a key to better understand observed phenomena in terms of CS.

\section{Results}

A network can be represented by a weighted digraph (or directed graph), defined by a vertex set $\mathcal{V}=\left\{v_{1}, \ldots, v_{N}\right\}$ (representing the nodes), an arrow set $\mathcal{E}_{k}$ for each kind of link $(k=1, \ldots, L)$, and a weight set $\mathcal{W}_{k}$ representing the connection weights for the $k$ th kind of link. The nodes can be of $M$ different kinds.

The weighted adjacency matrix $A^{k}$ embeds information about the arrow set $\mathcal{E}_{k}$, and the weight set $\mathcal{W}_{k}: A_{i j}^{k} \in \mathcal{W}_{k}$ is the (nonzero) weight of the link going from $v_{j}$ to $v_{i}$; $A_{i j}^{k}=0$ if there is no arrow from $v_{j}$ to $v_{i}$ and $A_{i j}^{k}$ belongs to $\mathcal{W}_{k}$ otherwise. In an undirected network, if there is an arrow with a certain weight going from $v_{i}$ to $v_{j}$ then there is also an arrow with the same weight going from $v_{j}$ to $v_{i}$; in this case, $A^{k}$ is a symmetric matrix and the underlying graph is undirected.

A multilayer network is characterized by the presence of different types of nodes interacting through different types of connections, with each layer being formed of nodes of the same type and/or connections of the same type ${ }^{31,32}$. In particular, in this paper, a layer is given by nodes of the same type ${ }^{23}$. Arrows can be inside each layer (intralayer connections) or go from layer to layer (interlayer connections).

The above definitions are illustrated through the network shown in Fig. 1.

We now associate a dynamics to each node, by considering a network with $N$ nodes, which are connected through $L$ different kinds of links. We model networks of this kind through the following set of dynamical equations $(i=1, \ldots, N)^{33}$, which provides a rather general description of deterministic systems governed by pairwise interactions

$$
\dot{\boldsymbol{x}}_{i}=\boldsymbol{f}_{i}\left(\boldsymbol{x}_{i}(t)\right)+\sum_{k=1}^{L} \sigma_{k} \sum_{j=1}^{N} A_{i j}^{k} \boldsymbol{h}^{k}\left(\boldsymbol{x}_{i}(t), \boldsymbol{x}_{j}\left(t-\delta_{k}\right)\right),
$$

where $\boldsymbol{x}_{i} \in \mathbb{R}^{n}$ is the $n$-dimensional state vector of the $i$ th node, $f_{i}: \mathbb{R}^{n} \rightarrow \mathbb{R}^{n}$ is the vector field of the isolated ith node describing the uncoupled dynamics of $\boldsymbol{x}_{i}, \sigma_{k} \in \mathbb{R}$ is the coupling strength of the $k$ th kind of link, $A^{k}$ is the weighted adjacency matrix with respect to the $k$ th kind of link, for which the pairwise interaction between two generic nodes $i$ and $j$ is described by the nonlinear function $\boldsymbol{h}^{k}: \mathbb{R}^{n} \times \mathbb{R}^{n} \rightarrow \mathbb{R}^{n}$, and $\delta_{k}$ is the transmission delay characteristic of the $k$ th kind of link. We assume that 

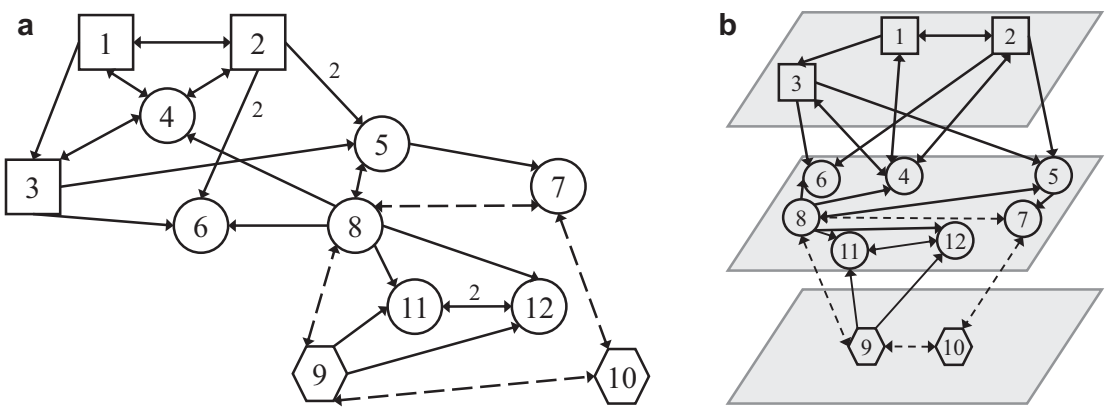

Fig. 1 Directed multilayer network. a Directed network with $N=12$ nodes of $M=3$ shape-coded different kinds and links of $L=2$ different kinds, represented by either solid or dashed arrows. The vertex set is $\mathcal{V}=\{1, \ldots, 12\}$, the arrow sets are $\mathcal{E}_{1}=\{(1,2),(1,3),(1,4),(2,1),(2,4),(2,5),(2,6), \ldots\}$ (solid arrows) and $\mathcal{E}_{2}=\{(7,8),(7,10),(8,7),(8,9), \ldots\}$ (dashed arrows). All weights in the sets $\mathcal{W}_{1}$ and $\mathcal{W}_{2}$ are equal to 1 except for the edges $(2,5)$, $(2,6),(11,12)$, and $(12,11)$ (all belonging to $\mathcal{E}_{1}$ ) that have weight equal to 2 . $\mathbf{b}$ The three layers for the network in (a): top layer with square nodes, middle layer with circle nodes, and bottom layer with hexagon nodes. Examples of intralayer connections: $1 \rightarrow 2$ (top layer), $8 \leftrightarrow 5$ (middle layer), $9 \leftrightarrow 10$ (bottom layer). Examples of interlayer connections: $2 \rightarrow 5$ (between top and middle layers), $8 \leftrightarrow 9$ (between middle and bottom layers).

each individual node can be of one out of $M$ different types (with $M \leq N): f_{i}(x)=f_{j}(x)$ if $i$ and $j$ are of the same type, $f_{i}(x) \neq f_{j}(x)$ otherwise. Within this general framework, where all oscillators can be different, if $M<<N$ the vector fields $f_{i}$ are not all different, but belong to a restricted set of $M$ models. Equation (1) describes the dynamics of a directed multilayer network with both nodes of different types and arrows of different types. The interpretation of Eq. (1) in terms of layers is made explicit in the Supplementary Note 1.

This paper deals with CS of the system (Eq. (1)), i.e., $\boldsymbol{x}_{i}(t)=$ $\boldsymbol{x}_{j}(t)$ for $i$ and $j$ that belong to the same cluster.

Existence. The existence of a synchronized cluster can be either ruled out or not, based on the analysis of the network topology and of the isolated node vector fields $f_{i}$. By a partition $\mathcal{P}=$ $\left\{C_{1}, C_{2}, \ldots, C_{Q}\right\}$ of a graph, we mean a partition of its vertex set into clusters, which satisfies the following properties: $\mathcal{P}=\left\{C_{i} \subset \mathcal{V}: C_{i} \cap C_{j}=\emptyset \forall i \neq j, \bigcup_{i=1}^{Q} C_{i}=\mathcal{V}\right\}$, where we call $C_{i}$ the $i$ th cluster, with $i=1, \ldots, Q$. We can identify each cluster through the labels of its constituting vertices and a given color. A cluster is said to be trivial if it consists of only one node.

Let $N_{i}$ be the number of nodes belonging to the cluster $C_{i}$. Therefore, $\sum_{i=1}^{Q} N_{i}=N$.

Each graph can admit different partitions, corresponding to different colorings of the graph. Invariant partitions, also called balanced partitions or equitable partitions, have a large number of applications in many fields such as graph, network, and control theories (see ref. ${ }^{34}$ and references therein). An invariant partition is one in which nodes with the same color follow the same time evolutions. Consequently, a partition (and the corresponding coloring) is balanced/equitable if all nodes with color $p$ have the same vector field $f_{i}$ and get the same overall input with delay $\delta_{k}$ from the nodes of color $q$, for $p, q=1, \ldots, Q$ and for any delay $\delta_{k}$ with $k=1, \ldots, L$, namely if

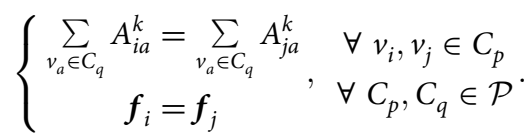

The existence of a balanced/equitable partition (coloring) for a given network is a necessary condition for the existence of a CS solution ${ }^{16}$.

In particular, among all possible equitable partitions, we focus on the partition $\mathcal{P}$ that corresponds to the so-called minimal balanced coloring, namely a balanced coloring with the minimal number of colors ${ }^{35-37}$. We also remark that the minimal balanced coloring provides the cluster configuration with the minimum number $Q$ of clusters.

Figure 2 shows the seven balanced partitions of a simple singlelayer directed network, whose adjacency matrix is reported in the Supplementary Note 2. The partition-labeled $\mathcal{P}$ corresponds to the minimal balanced coloring. The other partitions (labeled $\mathcal{P}^{j}$ ) correspond to non-minimal balanced colorings and are composed of clusters $C_{i}^{j}$. In particular, $\mathcal{P}^{6}$ contains only trivial clusters. It is easy to check that the partitions in Fig. 2 are all of the balanced partitions for this network.

Each balanced partition corresponds to a cluster-synchronous solution where all the nodes in the same cluster follow the same time evolution, but nodes in different clusters do not. These solutions can be expressed in terms of the so-called invariant synchrony subspaces (ISS), i.e., the polydiagonal subspaces under all admissible vector fields ${ }^{19,34,38-41}$.

The ISS $\Delta^{j}$ corresponding to each partition $\mathcal{P}^{j}$ of Fig. 2 can be easily determined by inspection: $\Delta^{1}$ is such that the state evolution of nodes 4 and 5 is the same, i.e., $\left\{\boldsymbol{x}_{4}=\boldsymbol{x}_{5}\right\} ; \Delta^{2}$ is such that $\left\{\boldsymbol{x}_{1}=\boldsymbol{x}_{2}\right.$ and $\left.\boldsymbol{x}_{3}=\boldsymbol{x}_{4}\right\} ; \Delta^{6}$ is such that each node of the network has a different time evolution, and so forth. A number of algorithms are available to compute all the ISS for a generic network (see "Methods").

We remark that not all the balanced partitions compatible with a given topology can also be observed when taking into account the network dynamics (1), as some of these may be unstable. Thus the dynamics governing the network must be incorporated in the study of stability for the CS solution.

Stability. The possibility of observing a synchronized cluster in a given physical system depends on its stability, and hence on the particular network structure and dynamics of the nodes.

Once the $Q$ equitable clusters corresponding to a balanced partition are found, the CS analysis can be focused on a simplified dynamical model (called quotient network) whose $Q$ nodes correspond to each one of the clusters. In particular, we analyze the stability of clusters by linearizing Eq. (1) about a state corresponding to exact synchronization among all the nodes within each cluster ${ }^{33}$. The Master Stability Function approach ${ }^{42}$, which evaluates the stability of synchronized solutions through linearization, is based on two standard steps: (i) finding the variational equations of the network about the synchronized solutions and (ii) expressing these variational equations in a new system of coordinates, which decouples the perturbation dynamics along the transverse manifold from that along the synchronous manifold. 


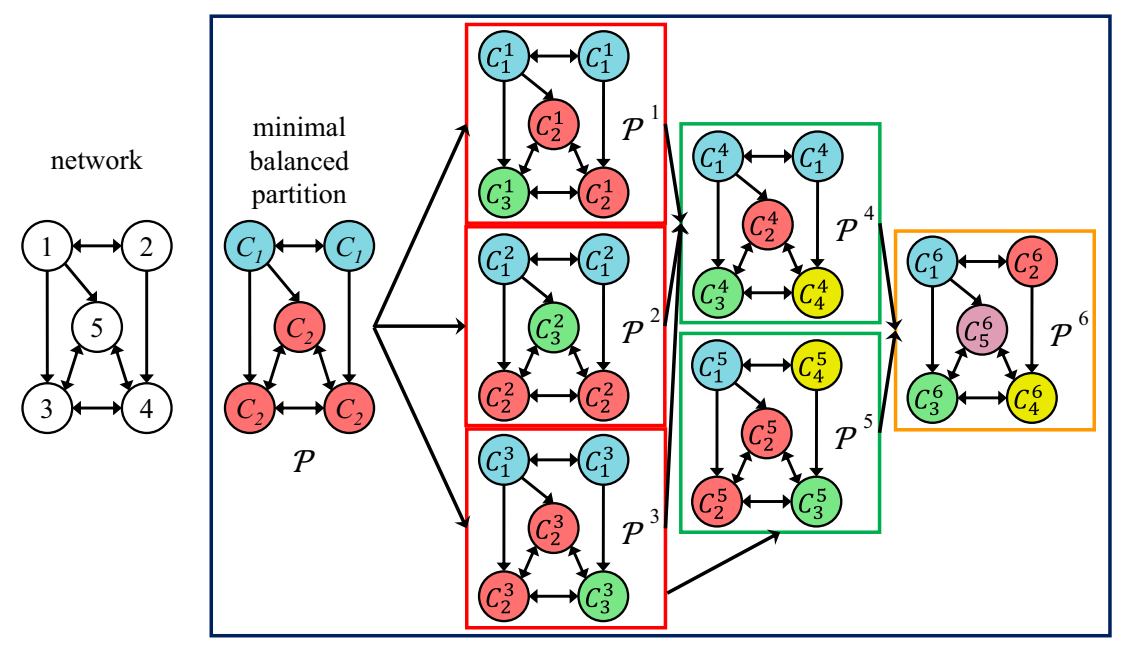

Fig. 2 Illustrative example with all balanced partitions. Balanced partitions (within the dark blue box) of a simple directed network with $N=5$ nodes. The partition $\mathcal{P}$ corresponds to the minimal balanced coloring, with two clusters $C_{1}$ (containing nodes 1 and 2 , colored blue) and $C_{2}$ (containing nodes $3-5$, colored red): each node of cluster $C_{2}$ receives one arrow from one node of $C_{1}$; each node of cluster $C_{1}$ receives one arrow from one node of $C_{1}$ and two arrows from nodes of $C_{2}$. Breaking these clusters, we can obtain all the other balanced partitions, which are non-minimal, because they contain a higher number of clusters. Each partition $\mathcal{P}^{1}, \mathcal{P}^{2}$, and $\mathcal{P}^{3}$ (within red boxes) contains three clusters, which are obtained from $\mathcal{P}$ by breaking the largest cluster into two smaller ones. For instance, $\mathcal{P}^{1}$ contains $C_{1}^{1}$ (with nodes 1 and 2 , colored blue), $C_{2}^{1}$ (with nodes 4 and 5 , colored red), and $C_{3}^{1}$ (with node 3 , colored green). This partition is balanced, because each node of $C_{1}^{1}$ receives one arrow from one blue node, each node of $C_{2}^{1}$ receives one arrow from a blue node, one arrow from a red node and one from a green node, and each node of $C_{3}^{1}$ receives one arrow from a blue node and two arrows from red nodes. Similarly, partitions $\mathcal{P}^{4}$ and $\mathcal{P}^{5}$ (within green boxes) contain four clusters each and are obtained from the partitions with three clusters by breaking the largest cluster into two smaller clusters: the partition $\mathcal{P}^{4}$ is obtained by breaking the red clusters of $\mathcal{P}^{1}, \mathcal{P}^{2}$, or $\mathcal{P}^{3}$; the partition $\mathcal{P}^{5}$ is obtained by breaking the blue cluster of $\mathcal{P}^{3}$. Finally, the partition $\mathcal{P}^{6}$ (within orange box) contains five trivial clusters (each one includes only one node).

We collect all state trajectories in the vector $x(t)=$ $\left[\boldsymbol{x}_{1}^{T}(t), \boldsymbol{x}_{2}^{T}(t), \ldots, \boldsymbol{x}_{N}^{T}(t)\right]^{T}$. As it is possible for all the nodes within a cluster to synchronize, we define the $q$ th cluster state: $\boldsymbol{s}_{q}(t)=$ $\boldsymbol{x}_{i}(t)$ for all $i$ in cluster $C_{q}$. Correspondingly, the network can produce $Q$ distinct synchronized motions $\left\{\boldsymbol{s}_{1}(t), \boldsymbol{s}_{2}(t), \ldots, \boldsymbol{s}_{Q}(t)\right\}$, one per cluster. We collect them in the vector $\boldsymbol{s}(t)=$ $\left[\boldsymbol{s}_{1}^{T}(t), \boldsymbol{s}_{2}^{T}(t), \ldots, \boldsymbol{s}_{Q}^{T}(t)\right]^{T}$. We then analyze the dynamics of a small perturbation $\boldsymbol{w}_{i}(t)=\boldsymbol{x}_{i}(t)-\boldsymbol{s}_{q_{i}}(t)(i=1, \ldots, N)$, where $\boldsymbol{s}_{q_{i}}(t)$ is the cluster state for node $i$ in cluster $C_{q}$, by linearizing around a specific network solution $\boldsymbol{s}(t)$.

In order to reduce the dimensionality of the stability analysis, we first divide the full state space of the variational equation into minimal flow-invariant subspaces and then calculate the maximum Lyapunov exponent (MLE) in each subspace, in order to determine whether perturbations within that subspace grow or decay. A key aspect for an efficient analysis of the stability of arbitrary synchronization patterns ${ }^{18,24,43}$ is to be able to optimally decouple the perturbation modes. To this end, we make a change of coordinates through the transformation matrix $T$, which maps the perturbation vector $\boldsymbol{w}=\left[\boldsymbol{w}_{1}^{T}, \boldsymbol{w}_{2}^{T}, \ldots, \boldsymbol{w}_{N}^{T}\right]^{T}$ into a new coordinate system $\boldsymbol{\eta}=\left[\boldsymbol{\eta}_{1}^{T}, \boldsymbol{\eta}_{2}^{T}, \ldots, \boldsymbol{\eta}_{N}^{T}\right]^{T}=\left(T \otimes \mathbb{I}_{n}\right) \boldsymbol{w}$, where the symbol $\otimes$ denotes the tensor product of matrices, i.e., the Kronecker product. The $N \times N$ matrix $T$ is made up of two parts: $T=\left[\begin{array}{c}T_{\|} \\ T_{\perp}\end{array}\right]$, where the $Q \times N$ submatrix $T_{\|}$is concerned with the directions inside the synchronization manifold and the corresponding perturbations do not influence the stability of the synchronized clusters; on the other hand, the $(N-Q) \times N$ submatrix $T_{\perp}$ is concerned with the directions transverse to the synchronization manifold and the evolution of the variational equation along these directions determines the stability of the synchronized clusters. Therefore, the perturbation dynamics are separated into two parts: that along the synchronous manifold (described by the first $Q$ components $\boldsymbol{\eta}_{i}$ ), which accounts for the stationary/periodic/chaotic state dynamics within each cluster and that transverse to it (described by the last components $\boldsymbol{\eta}_{i}, i \in[Q+$ $1, N]$ ), which accounts for the stability of clusters.

In this perspective, the coordinate system $\boldsymbol{\eta}$ must separate the evolution of transverse and parallel perturbation modes, by decoupling the transverse perturbation modes as much as possible. In particular, the entries of the $q$ th row of $T_{\|}$are all zero, except for those whose column index denotes one of the $N_{q}$ nodes belonging to $C_{q}$. The value of the nonzero entries is $1 / \sqrt{N_{q}}$.

On the other hand, $T_{\perp}$ does not have a unique structure but must satisfy two constraints:

- (A) $T_{\perp}$ contains $N_{q}-1$ rows for each cluster $C_{q}$; the $N$ entries of each row are are all zero, with the exception of those whose column index denotes one of the $N_{q}$ nodes belonging to $C_{q}$;

- (B) each row of $T_{\perp}$ is orthonormal to any other row of $T$.

The matrix $T$ transforms the coupling matrices $A^{k}$ into matrices $B^{k}=T A^{k} T^{-1}$. The system of equations governing the dynamics of the perturbation vector $\boldsymbol{w}$ is reducible, because all the adjacency matrices $A^{k}$ can be put in block-upper triangular form $B^{k}=T A^{k} T^{-1}$ by application of the same invertible transformation matrix $T$, where each matrix $B^{k}$ has the block structure

$$
B^{k}=\left[\begin{array}{cc}
B_{11}^{k} & B_{12}^{k} \\
0 & B_{22}^{k}
\end{array}\right]
$$

for any $k$. Note that this corresponds to effectively decoupling the perturbation vector into two parts, where the second one (containing the transverse perturbation modes) evolves independently of the first one (containing the perturbations inside the synchronization manifold). Among the many possible matrices $T$, 


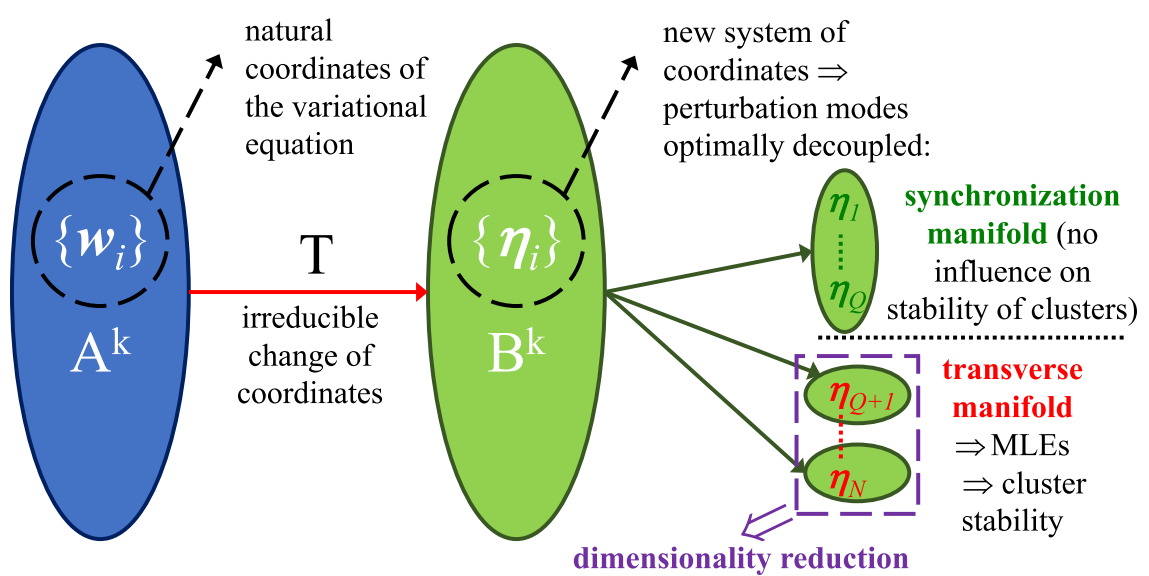

Fig. 3 Dimensionality reduction based on the transformation matrix $\mathbf{T}$. First (blue oval), the system is linearized about a synchronous solution $\mathbf{s}(t)$ (composed of $Q$ distinct synchronized motions), in order to analyze the dynamics of a small perturbation $\boldsymbol{w}_{i}(t)$ around each node $i(i=1, \ldots, N)$; the topology of each network layer is described by an adjacency matrix $A^{k}$. Second (large green oval), the transformed system is described by a new set of coordinates $\left\{\boldsymbol{\eta}_{i}\right\}$, which are coupled through the matrices $B^{k}=T A^{k} T^{-1}$. The new coordinates are decoupled into two sets (small green ovals), one containing the perturbations lying on the synchronization manifold, which do not affect the stability of the synchronized clusters, and one containing the perturbations transverse to the synchronization manifold, which affect the stability of the synchronized clusters. In particular, we look for the irreducible change of coordinates (and corresponding matrix $T$ ), which decouples the transverse perturbation modes as much as possible (green ovals within the dashed purple box). The transverse perturbation modes contain information on the stability of clusters, also evidencing the presence of interdependencies between different clusters.

we look for the one that ensures the maximal separation of the transverse perturbation modes.

A coordinate system $\boldsymbol{\eta}$ is no longer reducible (i.e., it is irreducible), if it is impossible to further separate the transverse perturbation modes $\boldsymbol{\eta}_{i}$ by application of another transformation matrix $T$. We then call irreducible the corresponding set of matrices $B^{k}$ that cannot be reduced further.

In the case of undirected networks, we can find a transformation matrix $T$ such that $B_{12}^{k}=0$ (null block) for any $k$. Therefore, each matrix $B^{k}$ can be reported in block-diagonal form; moreover, there are methods-based on either group theory $22,23,44$ or matrix algebras ${ }^{24,43}$ - that transform the matrix $B$ in an irreducible form.

In the case of directed networks, the matrix $B_{12}^{k}$ is not a null block, in general. Therefore, each matrix $B^{k}$ can be reported in block-upper triangular form ${ }^{27,33}$, but, at the best of our knowledge, there are no methods to find the matrix $T$ that transforms the perturbation modes in an irreducible form. We remark that a key requirement is that the matrix is decomposed so as to separate perturbations along the synchronous manifold from those along the transverse manifold. This prevents the use of standard matrix decomposition techniques, which do not take this into account.

We aim to find the matrix $T$ that converts the node coordinate system into the irreducible coordinate system, thus evidencing the interdependencies among the perturbation components in synchronized clusters of directed networks. Our proposal of a method to build up this matrix for directed networks is the major contribution of this paper.

The process for optimally decoupling the perturbation modes described above is summarized in Fig. 3.

One-way-dependent clusters and breaking vectors. As stated in "Introduction," in undirected networks, two clusters $C_{1}$ and $C_{2}$ are said to be intertwined ${ }^{18,27}$ if desynchronization of cluster $C_{1}$ can lead to loss of synchrony in cluster $C_{2}$ and vice versa. In directed networks, instead, it may happen that the stability of $C_{1}$ depends on the stability of $C_{2}$ but the opposite is not necessarily true. In this case, we say that $C_{1}$ is one-way-dependent on $C_{2}$.
For instance, the equitable partition $\mathcal{P}$ in Fig. 2 contains two clusters, $C_{1}=\{1,2\}$ and $C_{2}=\{3,4,5\}$. The stability of $C_{1}$ influences also the stability of $C_{2}$ : if only $C_{1}$ desynchronizes, then its nodes have no longer the same color and therefore also the nodes of $C_{2}$ cannot have the same color, which corresponds to either partition/coloring $\mathcal{P}^{1}$ or $\mathcal{P}^{2}$. On the other hand, if only $C_{2}$ desynchronizes, it is possible for the nodes of $C_{1}$ in all the corresponding balanced partitions $\left(\mathcal{P}^{3}, \mathcal{P}^{4}, \mathcal{P}^{5}, \mathcal{P}^{6}\right)$ to keep the same color. Therefore $C_{2}$ is one-way-dependent on $C_{1}$.

Our strategy to check for the presence of one-way-dependent clusters-also when the network has a large number of nodes and one cannot easily detect the interdependencies between clusters by inspection-is based on the ISS. We consider an equitable cluster belonging to the partition $\mathcal{P}$ : any other balanced partition $\mathcal{P}^{j}$ can be obtained by breaking one or more clusters of $\mathcal{P}$ into the subclusters $C_{i}^{j}$.

We consider the minimal balanced partition $\mathcal{P}$, the non-minimal balanced partition $\mathcal{P}^{j}$, and the $i$ th cluster $C_{i}^{j} \in \mathcal{P}^{j}$. Let $\mathcal{B}_{q}^{j}$ be the $N$ length row vector describing the transition from the cluster $C_{q} \in \mathcal{P}$ to smaller clusters $C_{i}^{j} \in \mathcal{P}^{j}$. We label $\mathcal{B}_{q}^{j}$ so that the superscript $j$ indicates the partition $\mathcal{P}^{j}$ and the subscript $q$ indicates the specific cluster, which is broken. Henceforth, we will call each of these vectors a breaking vector. The entries of $\mathcal{B}_{q}^{j}$ corresponding to nodes that do not belong to $C_{q}$ are set to 0 , whereas those corresponding to nodes belonging to $C_{q}$ have the same value if they belong to the same cluster $C_{i}^{j}$. Notice that the pair $q, j$ is meaningful only if $C_{q}$ breaks in the transition from $\mathcal{P}$ to $\mathcal{P}^{j}$. For instance, with reference to Fig. 2, if we consider $\mathcal{P}^{1}$ and cluster $C_{2} \in \mathcal{P}$, the breaking vector $\mathcal{B}_{2}^{1}$ has the following structure: $\mathcal{B}_{2}^{1}=[\underbrace{\begin{array}{ll}0 & 0\end{array}}_{C_{1}} \underbrace{\overbrace{a}^{C_{2}^{1}} \overbrace{b}^{C_{3}^{1}}}_{C_{2}}]$. By contrast, the breaking vector $\mathcal{B}_{1}^{4}$ is meaningless, since $C_{1}$ does not break in the transition from $\mathcal{P}$ to $\mathcal{P}^{4}$.

We define the intertwining index $n_{q}^{j}$ as the number of clusters $C_{p} \in \mathcal{P}$ other than $C_{q}$ (i.e., $p \neq q$ ) which are broken after breaking 
cluster $C_{q}$ as described by the vector $\mathcal{B}_{q}^{j}$. In other words, $n_{q}^{j}$ is the number of clusters of $\mathcal{P}$ (in addition to $C_{q}$ ), which are affected by the breaking action of $\mathcal{B}_{q}^{j}$, namely the number of other clusters of $\mathcal{P}$ whose stability depends on the desynchronization of $C_{q}$. For instance, in the example of Fig. $2, n_{2}^{1}=0$, because $C_{1}$ remains unaltered in the transition from $\mathcal{P}$ to $\mathcal{P}^{1}$ (notice that in $\mathcal{P}^{1} C_{1}$ is labeled $C_{1}^{1}$ ). This means that the loss of stability (i.e., the breaking) of $C_{2}$ does not influence any other cluster. By contrast, $n_{1}^{5}=1$, because in the transition from $\mathcal{P}$ to $\mathcal{P}^{5}$ or to $\mathcal{P}^{6}$ also $C_{2}$ is broken by $\mathcal{B}_{1}^{5}$. This means that the breaking of $C_{1}$ influences the stability of $C_{2}$, therefore $C_{2}$ is one-way-dependent on $C_{1}$.

The breaking vectors play a fundamental role not only in checking the presence of one-way-dependent clusters, but also in finding the irreducible transformation for directed networks, as described in the following.

Constructive method to obtain an irreducible transformation for directed networks. As stated above, we aim to find the matrix $T$ that converts the node coordinate system to the irreducible coordinate system, thus evidencing the interdependencies among the perturbation components.

For undirected networks, this matrix can be found as described in refs. ${ }^{22-24}$. To the best of our knowledge, there is no general method for finding this transformation matrix for directed networks; the method proposed in ref. ${ }^{27}$ allows calculation of a matrix $T$, but in general it does not correspond to an irreducible transformation. Here we propose a method that outputs the irreducible transformation for directed networks and holds also (as a particular case) for undirected networks.

The key variational equation for studying cluster stability has the following structure ${ }^{33}$ :

$$
\dot{\boldsymbol{\eta}}=\Psi_{1}[\boldsymbol{s}(t)] \boldsymbol{\eta}(t)+\sum_{k=1}^{L} \Psi_{2}^{k}\left[\boldsymbol{s}\left(t-\delta_{k}\right)\right] \boldsymbol{\eta}\left(t-\delta_{k}\right),
$$

where $\Psi_{1}$ and $\Psi_{2}^{k}$ are time-dependent matrices that depend on the synchronous solution $\boldsymbol{s}(t)$.

The block-diagonal matrix $\Psi_{1}$ depends only on the network (not on the coordinate change) and, through its action, $\dot{\boldsymbol{\eta}}_{j}$ only depends on $\boldsymbol{\eta}_{\boldsymbol{j}}$. By contrast, each matrix $\Psi_{2}^{k}$ depends on the transformation matrix $T$ through a matrix $B^{k}$ and therefore $\dot{\eta}_{j}$ is related to the other perturbation components by the second term on the right-hand side of Eq. (4). Taking into account that $T$ is an orthogonal matrix (i.e., $T^{-1}=T^{T}$ ), each matrix $B^{k}$ has the following structure:

$B^{k}=T A^{k} T^{-1}=\left[\begin{array}{c}T_{\|} \\ T_{\perp}\end{array}\right] A^{k}\left[\begin{array}{cc}T_{\|}^{T} & T_{\perp}^{T}\end{array}\right]=\left[\begin{array}{c:c}T_{\|} A^{k} T_{\|}^{T} & T_{\|} A^{k} T_{\perp}^{T} \\ \hdashline T_{\perp} A^{k} T_{\|}^{T_{\|}} & T_{\perp} A^{k} T_{\perp}^{T^{-}}\end{array}\right]$,

where the block $T_{\perp} A^{k} T_{\|}^{T}$ contains only 0 entries (the proof of this statement is in the section "Methods"), whereas the block $T_{\|} A^{k} T_{\perp}^{T}$ is null for undirected networks and contains nonzero entries for directed networks ${ }^{33}$. Therefore, in general, $B^{k}$ can be written as

$B^{k}=\left[\begin{array}{c:c}B_{\|}^{k} \\ \hdashline 0 & B_{\perp}^{k-}\end{array}\right]$

This implies that the transverse perturbations $\left(\boldsymbol{\eta}_{Q+1}, \ldots, \boldsymbol{\eta}_{N}\right)$ accounting for cluster stability do not depend on the parallel perturbations $\left(\boldsymbol{\eta}_{1}, \ldots, \boldsymbol{\eta}_{Q}\right)$ along the synchronous manifold.

In particular, our main goal is to analyze CS by decoupling the transverse perturbations as much as possible, with the goal of reducing the dimensionality of the problem. Therefore, we look for the matrix $T$ that transforms the matrix $B_{\perp}=\sum_{k=1}^{L} B_{\perp}^{k}$ in an irreducible block-diagonal form with each block appearing as a block-upper triangular matrix whenever possible. The method to
Table 1 Breaking vectors and intertwining indices.

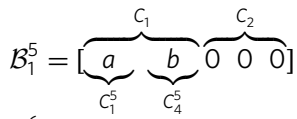

$$
\begin{aligned}
& \mathcal{B}_{1}^{6}=[\underbrace{a}_{c_{1}^{6}} \underbrace{b}_{c_{2}^{6}} \quad 0 \quad 00]
\end{aligned}
$$

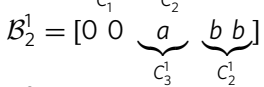

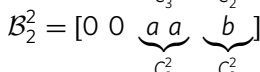

$$
\begin{aligned}
& \mathcal{B}_{2}^{3}=\left[\begin{array}{llll}
0 & 0 & a & b
\end{array}\right]
\end{aligned}
$$

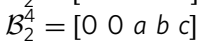

$$
\begin{aligned}
& \mathcal{B}_{2}^{5}=\left[\begin{array}{llll}
0 & 0 & a & b
\end{array}\right]
\end{aligned}
$$

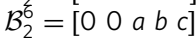

$$
\begin{aligned}
& n_{1}^{5}=1 \\
& n_{1}^{6}=1 \\
& n_{2}^{1}=0 \\
& n_{2}^{2}=0 \\
& n_{2}^{3}=0 \\
& n_{2}^{4}=0 \\
& n_{2}^{5}=1 \\
& n_{2}^{6}=1
\end{aligned}
$$

Breaking vectors $\mathcal{B}_{q}^{j}$ and corresponding intertwining indices $n_{q}^{j}$ for each partition $\mathcal{P}^{j}$ and for the equitable clusters $C_{1}$ and $C_{2}$ of the network of Fig. 2, partition $\mathcal{P}$. For the first four breaking vectors also the broken clusters are shown. Cluster $C_{1}$ is broken only in partitions $\mathcal{P}^{5}$ and $\mathcal{P}^{6}$ (see the first two rows and Fig. 2). In $\mathcal{P}^{5}$ (see Fig. 2), $C_{1}$ is broken as described in the first row $\left(\mathcal{B}_{1}^{5}\right)$ and also $C_{2}$ is broken, therefore $n_{1}^{5}=1$. Cluster $C_{2}$ is broken in all partitions. In particular, in $\mathcal{P}^{1}$ cluster, $C_{2}$ is broken as described in the third row $\left(\mathcal{B}_{2}^{1}\right)$ while cluster $C_{1}$ is not broken (see Fig. 2), therefore $n_{2}^{1}=0$.

construct the matrix $T$ with these characteristics is based on the breaking vectors, as we explain below.

The choice of the $T$ rows related to the equitable cluster $C_{q}$ does not depend on the other clusters: as a matter of fact, owing to the constraints (A) and (B), the rows of $T$ related to a cluster are orthonormal to those related to any other cluster.

We construct the matrix $T$ by stacking together $Q$ matrices $T_{q}$ (one for each cluster), with dimensions $N_{q} \times N$. The first row of each matrix $T_{q}$ has entry $1 / \sqrt{N_{q}}$ for each column corresponding to a node in $C_{q}$, zero otherwise. The other $N_{q}-1$ rows are taken among the vectors $\mathcal{B}_{q}^{j}$ such that (i) the rows of $T_{q}$ are orthonormal and (ii) $n_{q}^{j}$ is minimum. If there is more than one vector $\mathcal{B}_{q}^{j}$ fulfilling these conditions, we select the one with the largest number of occurrences.

Once we have all matrices $T_{q}(q=1, \ldots, Q)$,

- $T_{\|}$contains the first rows of all the matrices $T_{q}$;

- $T_{\perp}$ contains all the other rows of $T_{q}$, sorted in order to make the matrix $B_{\perp}$ block-diagonal with minimal block sizes and with sizes decreasing along the diagonal. This is done by applying the Cuthill-McKee algorithm ${ }^{45}$ to $B$ and associated swaps of rows and columns to $T$.

This choice ensures that $T$ is the transformation we are looking for, because $B_{\perp}$ is in a form that is as block-diagonal as possible (diagonal if $n_{q}^{j}=0$ for any $q$ and $j$ ).

To illustrate the method, we detail the construction of the matrix $T$ for the network of Fig. 2.

Table 1 shows the breaking vectors $\mathcal{B}_{q}^{j}$ and the corresponding intertwining indices $n_{q}^{j}$ for each partition $\mathcal{P}^{j}$ and for the equitable clusters $C_{1}$ and $C_{2}$ of partition $\mathcal{P}$ in Fig. 2.

The matrices $T_{q}(q=1,2)$ are built as follows. Here we detail the construction of the matrix $T_{2}$, by taking the following steps:

(1) We initialize $T_{2}$ with the row corresponding to the synchronous manifold: $T_{2}=1 / \sqrt{3}\left[\begin{array}{lllll}0 & 0 & 1 & 1 & 1\end{array}\right]$.

(2) Since there is more than one breaking vector fulfilling conditions (i) and (ii), among all the breaking vectors $\mathcal{B}_{2}^{\prime}$ with $n_{2}^{j}=0$, we select and append to $T_{2}$ the ones $\left(\mathcal{B}_{2}^{3}\right.$ and $\mathcal{B}_{2}^{4}$ ) whose structure appears more frequently (twice) in 

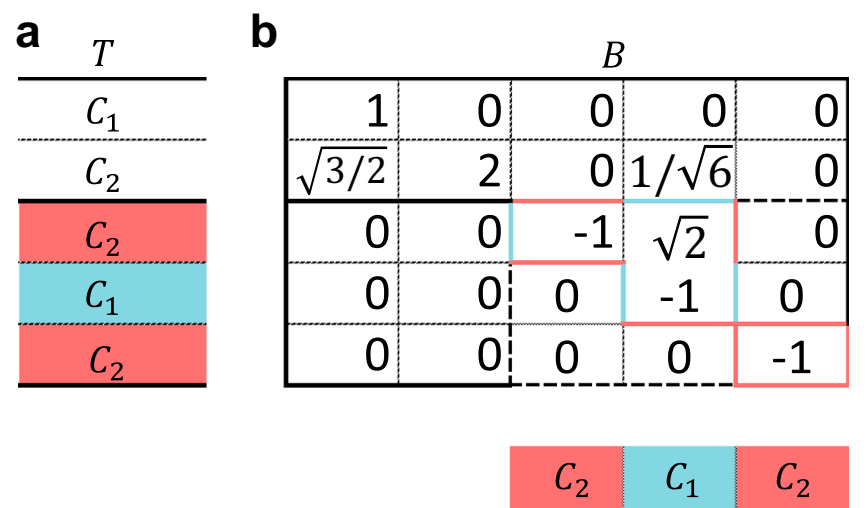

Fig. 4 Matrices $\boldsymbol{T}$ and $\boldsymbol{B}$ for the example of Fig. 2, partition $\mathcal{P}$. a Structure of the matrix $T$, with the rows of $T_{\perp}$ color-coded as the corresponding clusters. $\mathbf{b}$ Complete matrix $B$. The transverse block $B_{\perp}$ (within the dashed borders) has an upper triangular structure, containing two subblocks with nonzero entries (color-bordered blocks). The upper subblock (with red and blue borders) characterizes (see the corresponding rows in the $T$ structure) the perturbation affecting clusters $C_{2}$ (first row of the subblock), which depends on both clusters, and the one affecting $C_{1}$ (second row), which depends only on $C_{1}$ (single diagonal entry). The red scalar subblock evidences a second perturbation affecting cluster $C_{2}$, which is independent of cluster $C_{1}$. Therefore, $C_{1}$ is an independent cluster, whereas $C_{2}$ is oneway-dependent on $C_{1}$.

Table 1 and that are orthonormal to the other rows of $T_{2}$ :

$$
T_{2}=\left[\begin{array}{ccccc}
0 & 0 & \frac{1}{\sqrt{3}} & \frac{1}{\sqrt{3}} & \frac{1}{\sqrt{3}} \\
0 & 0 & -\frac{1}{\sqrt{6}} & \frac{2}{\sqrt{6}} & -\frac{1}{\sqrt{6}} \\
0 & 0 & -\frac{1}{\sqrt{2}} & 0 & \frac{1}{\sqrt{2}}
\end{array}\right]
$$

(3) Since $T_{2}$ has already the proper size $\left(N_{2}=3\right.$ rows), we stop here. In case this condition was not satisfied, we would need to consider the breaking vectors with larger intertwining indices $n_{2}^{j}$.

$T_{1}$ is obtained analogously and is as follows:

$$
T_{1}=\frac{1}{\sqrt{2}}\left[\begin{array}{ccccc}
1 & 1 & 0 & 0 & 0 \\
1 & -1 & 0 & 0 & 0
\end{array}\right]
$$

Now we can build up the matrix $T: T_{\|}$contains the first row of $T_{1}$ and the first row of $T_{2}, T_{\perp}$ contains all the other rows of $T_{1}$ and $T_{2}$. The rows of $T_{\perp}$ are sorted in order to make the matrix $B=$ $T A^{1} T^{T}$ as close as possible to a diagonal matrix:

$T=\left[\begin{array}{l}T_{\|} \\
\bar{T}_{\perp}\end{array}\right]=\left[\begin{array}{ccccc}\frac{1}{\sqrt{2}} & \frac{1}{\sqrt{2}} & 0 & 0 & 0 \\
0 & 0 & \frac{1}{\sqrt{3}} & \frac{1}{\sqrt{3}} & \frac{1}{\sqrt{3}} \\
\hdashline 0 & 0 & -\frac{1}{\sqrt{6}} & \frac{2}{\sqrt{6}} & -\frac{1}{\sqrt{6}} \\
-\frac{1}{\sqrt{2}} & \frac{1}{\sqrt{2}} & 0 & 0 & 0 \\
0 & 0 & -\frac{1}{\sqrt{2}} & 0 & \frac{1}{\sqrt{2}}\end{array}\right]$\begin{tabular}{l}
$\rightarrow C_{2}$ \\
$\rightarrow C_{2}$ \\
\hline
\end{tabular}

The corresponding matrix $B$ is shown in Fig. $4 \mathrm{~b}$. From the block-upper triangular structure of the transverse block $B_{\perp}$ in Fig. $4 \mathrm{~b}$, we see that the perturbation affecting cluster $C_{1}$ is independent of those affecting cluster $C_{2}$. One of the two perturbations affecting cluster $C_{2}$ is in turn independent of that affecting cluster $C_{1}$. Assuming that this perturbation is stable, the remaining perturbation affecting cluster $C_{2}$ is also independent. This reflects the fact that the stability of $C_{1}$ does not depend on the stability of $C_{2}$, whereas the stability of $C_{2}$ depends on the stability of $C_{1}$. Therefore, as stated above, $C_{2}$ is one-waydependent on $C_{1}$.

In summary (see the Supplementary Fig. 7), the characteristics of interdependence between clusters (intertwined, one-way dependent or independent) in the general class of networks (1) can be deduced from the ISS and from the breaking vectors; hence, they are based exclusively on the network topology. Through the matrix $B$ related to the irreducible transformation matrix $T$ built up as described above, we can evaluate the stability of clusters, thus extending the method proposed in ref. ${ }^{33}$ to a larger class of networks, which includes directed networks. In so doing, we solve an open problem ${ }^{24}$. Moreover, by using the irreducible transformation matrix $T$ based on the breaking vectors, one can easily detect the presence of intertwined or one-way-dependent clusters by inspecting the submatrix $B_{\perp}$ :

- each upper triangular block of the matrix $B_{\perp}$ indicates the presence of one-way-dependent clusters;

- each full block of the matrix $B_{\perp}$ indicates that the associated clusters are intertwined.

To better appreciate the advantages of detecting the interdependencies among clusters in more complex networks, Fig. 5 shows the application of the method to the network of Fig. 1a, whose minimal balanced partition has $Q=5$ clusters.

In the following, the proposed method is applied to two examples.

Violin players. The first example is from ref. ${ }^{28}$ and is concerned with a human network of violin players executing a musical phrase. The coupling strength between two players is defined as the ratio between the sound volume of the coupled violin and the sound volume of the player's own violin, while maintaining constant the total volume that each player hears. The dynamics of the phase $\varphi_{i}$ of the $i$ th violin player is simulated according to the model proposed in ref. ${ }^{28}$

$$
\dot{\varphi}_{i}=\omega_{i}+\kappa \sum_{j=1}^{N} A_{i j} \sin \left(\varphi_{j}(t-\delta)-\varphi_{i}(t)\right)
$$

where $\omega_{i}$ is the eigenfrequency of the $i$ th player, $\kappa=0.2 \mathrm{~Hz}$ is the coupling strength and $\delta$ is the delay in seconds between the players. Each player can decide to ignore one input, in which case, a directed connection is established. Reference ${ }^{28}$ studies the formation of these directed network topologies as the delay $\delta$ is varied experimentally.

Unlike ref. ${ }^{28}$, where the eigenfrequencies were randomly distributed in the range $[0.25,0.3] \mathrm{Hz}$, here we consider all players $(i=1, \ldots, N)$ with the same eigenfrequency $\omega_{i}=0.25 \mathrm{~Hz}$. We focus on some of the ring topologies with $N=8$ violin players studied in ref. ${ }^{28}$. On the basis of the delay $\delta$, the experiments in ref. ${ }^{28}$ have determined that each player either pays attention to both closest neighbors or decides to ignore either one of them, which corresponds to effectively changing the network connectivity.

(a) Undirected network. For low delay (roughly, $\delta<1 \mathrm{~s}$ ), each player listens to both closest neighbors, which corresponds to having bidirectional connections, as shown in Fig. 6a. The corresponding minimal balanced coloring is uniform, meaning that the quotient network contains only one node (Fig. 6b) and thenetwork can synchronize globally. This synchronization pattern corresponds to the eight violins being played "in-phase" and is stable because the corresponding MLE is negative for any value of $\delta$.

Similarly, in the case of large delay $\delta \in[3,4]$ s, each player listens to both closest neighbors, thus leading again to global synchronization. However, an open question is whether the network in Fig. 6a can admit the "out-of-phase" synchronization experimentally found in ref. ${ }^{28}$. To this end, we consider the balanced coloring shown in Fig. 6c, corresponding to two clusters and to a quotient network of two nodes (Fig. 6d). Let $\Delta \varphi$ be the 

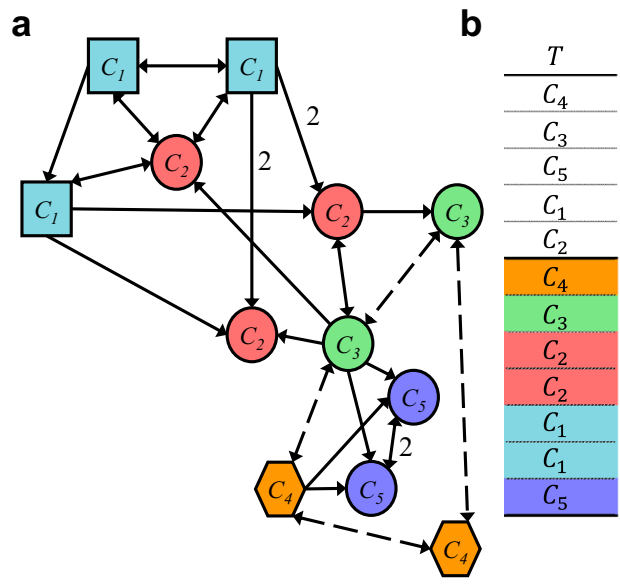

c

\begin{tabular}{|c|c|c|c|c|c|c|c|c|c|c|c|}
\hline \multicolumn{12}{|c|}{$\underline{B}$} \\
\hline 0 & 3 & 0 & 0 & $\sqrt{3 /}$ & $\sqrt{3 / 2}$ & 0 & 0 & 0 & 0 & 0 & 0 \\
\hline 0 & 0 & 0 & 0 & 0 & 0 & 0 & -1 & -1 & $-\sqrt{2}$ & $1 / \sqrt{6}$ & 0 \\
\hline 0 & 0 & 2 & 1 & 1 & 1 & -1 & 0 & 0 & 0 & 0 & 0 \\
\hline 0 & 0 & 2 & 1 & 1 & 1 & -1 & 0 & 0 & 0 & 0 & 0 \\
\hline$\sqrt{2 / 3}$ & 0 & 0 & 1 & 1 & 0 & 0 & $\sqrt{1 / 3}$ & $\sqrt{5 / 4}$ & 0 & 0 & 0 \\
\hline 0 & 0 & 0 & 0 & 0 & 1 & -1 & 0 & 0 & 0 & 0 & 0 \\
\hline 0 & 0 & 0 & 0 & 0 & -1 & 1 & 0 & 0 & 0 & 0 & 0 \\
\hline 0 & 0 & 0 & 0 & 0 & 0 & 0 & 0 & 0 & 0 & $\sqrt{2 / 3}$ & 0 \\
\hline 0 & 0 & 0 & 0 & 0 & 0 & 0 & 0 & 0 & 0 & $\sqrt{2 / 3}$ & 0 \\
\hline 0 & 0 & 0 & 0 & 0 & 0 & 0 & 0 & 0 & 0 & $\sqrt{1 / 3}$ & 0 \\
\hline 0 & 0 & 0 & 0 & 0 & 0 & 0 & 0 & 0 & 0 & -1 & 0 \\
\hline 0 & 0 & 0 & 0 & 0 & 0 & 0 & 0 & 0 & 0 & 0 & -2 \\
\hline
\end{tabular}

Fig. 5 Summarizing illustrative example. a The minimal balanced partition for the network of Fig. 1a, which has $Q=5$ clusters: $C_{1}=\{1,2,3\}$ (colored blue), $C_{2}=\{4,5,6\}$ (colored red), $C_{3}=\{7,8\}$ (colored green), $C_{4}=\{9,10\}$ (colored orange), and $C_{5}=\{11,12\}$ (colored purple). b Structure of the corresponding matrix $T$. The $T_{\perp}$ rows are color-coded as the corresponding clusters. c Complete matrix $B$. The transverse block $B_{\perp}$ (within the dashed borders) has an upper triangular structure, containing three irreducible subblocks (color-bordered blocks). The purple-bordered subblock characterizes the only perturbation affecting cluster $C_{5}$ (see the corresponding row in the $T$ structure), which is independent (single diagonal entry). The green-orangebordered $2 \times 2$ subblock evidence the two perturbations affecting clusters $C_{3}$ (second row of the block) and cluster $C_{4}$, which are intertwined. The blue-redbordered upper triangular subblock is concerned with the two perturbations affecting clusters $C_{1}$ and $C_{2}$ : both of them depend only on $C_{1}$, indicating that $C_{2}$ is one-way dependent on $C_{1}$.
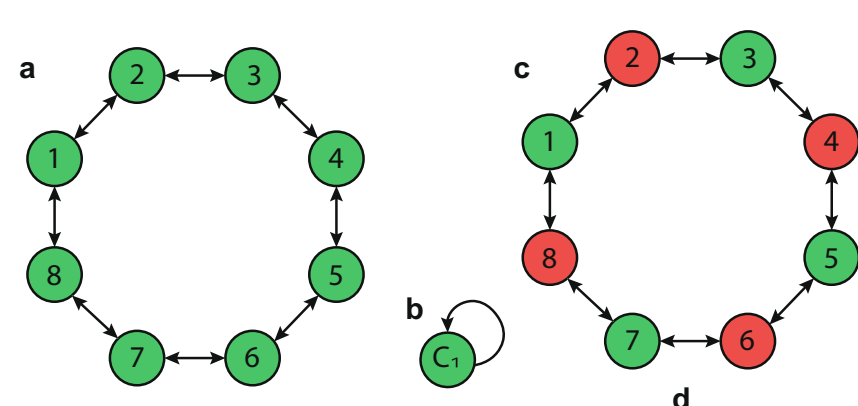

d
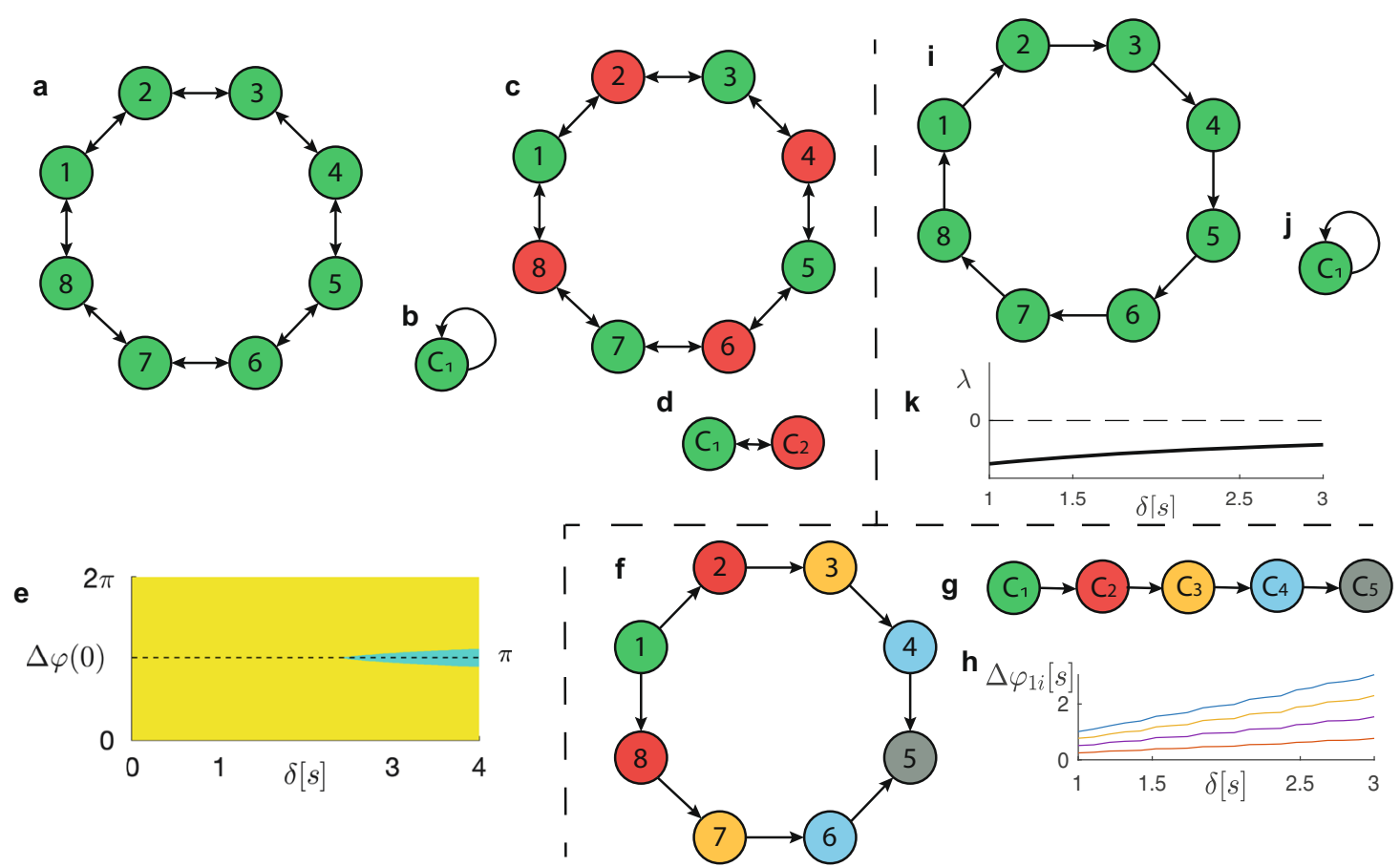

Fig. 6 Example 1: ring of 8 violin players. For delay $\delta<1 \mathrm{~s}$ or $3<\delta<4 \mathrm{~s}$, the network of violin players can be represented as undirected (a-d), and for $1 \mathrm{~s}<$ $\delta<3 \mathrm{~s}$ as a directed network with "arrowhead" topology, oriented either clockwise (as in $\mathbf{f}, \mathbf{g}, \mathbf{i}, \mathbf{j}$ ) or counterclockwise. a Undirected network with minimal balanced coloring ("in-phase" global synchronization pattern). b Corresponding quotient network. c Undirected network with (non-minimal) balanced coloring. d Corresponding quotient network. e Basins of attraction of the two stable solutions admitted by the quotient network (d): "in-phase" global synchronization (yellow region) and "out-of-phase" synchronization with two clusters oscillating in antiphase (green region). $\mathbf{f}$ Directed network with "arrowhead" topology and balanced coloring. $\mathbf{g}$ Corresponding quotient network. h Asymptotic phase differences $\Delta \varphi_{i 1}$ vs. $\delta$ : it is apparent that $\Delta \varphi_{i 1}$ grows linearly with $\delta$. i Directed network with "arrowhead" topology and minimal balanced coloring. $\mathbf{j}$ Corresponding quotient network. k MLE $\lambda$ corresponding to this synchronization pattern, showing stability as $\lambda(\delta)<0$ for any $\delta \in(1,3)$.

phase lag between the two clusters. For each $\delta$ value, the quotient network has two stable equilibrium points: one for $\Delta \varphi=0$ and one for $\Delta \varphi=\pi$. The first one corresponds to the global synchronization predicted by the minimal balanced coloring, whereas the second one corresponds to a stable out-of-phase synchronization with two clusters oscillating in antiphase. Figure 6e shows the pairs $(\delta, \Delta \varphi(0))$ that generate trajectories eventually approaching either one of the two equilibrium points: $\Delta \varphi=0$ (yellow points) and $\Delta \varphi=\pi$ (green points). The figure evidences the presence of multistability, which is in agreement 
with the analysis in ${ }^{28}$, even if antiphase synchronization was not found with the original model. We note that in ref. ${ }^{28}, \delta$ is increased linearly with time and the initial condition $\Delta(0)$ is therefore always close to 0 , thus preventing the network from reaching the experimentally found stable antiphase pattern. There are some quantitative discrepancies between ref. ${ }^{28}$ and our work, regarding the delay values at which the out-of-phase state of synchronization starts to be experimentally detected $(<2 \mathrm{~s})$. The quantitative differences between experimental and numerical results are mainly due to the simple model adopted in both papers. Further quantitative differences in the simulated results can be ascribed to two main changes in our modeling approach with respect to ref. ${ }^{28}$ : (i) we consider the asymptotic network state for a fixed delay, whereas ${ }^{28}$ considers a time-varying delay, namely the results contain also transient information; (ii) we consider the same oscillation frequency for all the oscillators, in order to allow for the existence of CS. We also remark that detection of multistability is harder in the original network, because the space of the phase lags is $\mathbb{R}^{7}$, whereas in the quotient network it is only $\mathbb{R}^{1}$, owing to the dimensionality reduction. Notice that for low $\delta$ the equilibrium point $\Delta \varphi=\pi$ is unstable, indicating that we can only have global synchronization of the violin players.

The matrix $B$ for this network is reported and analyzed in the Supplementary Note 3.1.

(b) Directed network. If $\delta \in[1,3] \mathrm{s}$, each violin player ignores one of its closest neighbors, leading to either one of two different directed networks. The first network is configured according to the "arrowhead" topology shown in Fig. 6f. The corresponding minimal balanced coloring contains five colors, therefore the network admits $Q=5$ clusters (see the quotient network in Fig. 6g), two of which are trivial, which excludes the possibility of global synchronization. The matrix $B$ for this network is reported and analyzed in the Supplementary Note 3.2. The corresponding clusters are stable. The phase difference $\Delta \varphi_{i j}=\varphi_{i}-\varphi_{j}$ between two clusters grows linearly with $\delta$, as shown in Fig. $6 \mathrm{~h}$ using node 1 as reference (i.e., $j=1$ ).

The second network has unidirectional connections with the same orientation (either clockwise or counterclockwise), which corresponds to the uniform balanced coloring in Fig. 6i and global synchronization associated with a rotational symmetry. Here we do not obtain the "vortex" pattern found in ref. ${ }^{28}$, due to our assumption of identical eigenfrequencies $\omega_{i}$ for all players.

Further details about the matrices obtained by applying our method are provided in Supplementary Data 1.

Neural activity and chimera states. The second example is adapted from ref. ${ }^{29}$. It is concerned with a network (shown in Fig. 7a) consisting of $N=20$ neurons, distributed in $M=2$ layers with the same number $N / 2$ of nodes and connected through $L=2$ kinds of synapses. The upper layer (layer I, in red) is composed of uncoupled identical neurons (see "Methods") and the lower layer (layer II, in blue) is composed of coupled identical neurons, different from those of layer I. Let $1, \ldots, N / 2$ be the labels of neurons of layer II and $N / 2+1, \ldots, N$ those of neurons in layer I. Each neuron $i=1, \ldots, N / 2$ of layer I is connected to neuron $i+$ $N / 2$ of layer II through a double directed chemical excitatory synapse with strength $\sigma_{2}$ in one direction (see thick red connections from layer II to layer I) and $\sigma_{2} / 4$ in the opposite direction (see thin red connections from layer I to layer II).

In layer II, each neuron is connected to its $P=6$ closest neighbors through identical electrical synapses (gap junctions) with strength $\sigma_{1}$. Nodes of layer I indirectly interact only through the layer II neurons.
The minimal balanced coloring of the network (Fig. 7b) evidences the presence of two clusters $C_{1}=\{1, \ldots, N / 2\}$ and $C_{2}=$ $\{N / 2+1, \ldots, N\}$, namely cluster $C_{1}$ contains all the neurons of layer I and cluster $C_{2}$ all those of layer II. The corresponding quotient network (Fig. 7c) is composed of two nodes only. Through the proposed method (see the Supplementary Note 3.3, for details), we obtain the matrix $T$ corresponding to the irreducible transformation of coordinates. The corresponding matrix $B$ shows that cluster $C_{2}$ is intertwined with $C_{1}$.

Following refs. 29,30 , we study the stability of clusters by varying the synaptic efficacy $\sigma_{2}$, with $\sigma_{1}=0.005$. Figure $7 \mathrm{~d}$ shows the MLEs $\Lambda_{q}$ corresponding to cluster $C_{q}(q=1,2)$ vs. $\sigma_{2}$ (overlapping curves, because the clusters are intertwined): it is evident that these clusters are stable only for $\sigma_{2}$ values larger than a threshold approximately equal to 1.5 .

Next we want to check the presence of chimera states 46,47 , which are characterized by the coexistence of coherent and incoherent dynamics. This kind of state has been detected in several neural systems and it has been associated to either pathological or normal activity ${ }^{48,49}$. For instance, during an epileptic seizure, some regions of the brain are strongly synchronized, whereas others are desynchronized ${ }^{50}$. Similarly, the two brain hemispheres of a sleeping dolphin have independently synchronized and desynchronized behaviors at the same time ${ }^{51}$ : one hemisphere is in sleep and another remains awake. This unihemispheric slow-wave sleep has also been found in other aquatic animals and migrating birds ${ }^{52}$.

The stable clusters $C_{1}$ and $C_{2}$ of Fig. $7 \mathrm{c}$ correspond to coherent dynamics. In order to check whether the considered network can generate other patterns of synchronization, we consider a balanced coloring that is not minimal.

The network admits many non-minimal balanced colorings, one of which is shown in Fig. 7e, containing a larger number of clusters $(Q=12)$, as pointed out by the corresponding quotient network (Fig. 7f). Also in this case, through the proposed method (see the Supplementary Note 3.3, for details), we obtain the matrix $T$ corresponding to the irreducible transformation of coordinates. Analysis of the corresponding matrix $B$ allows to conclude that all nontrivial clusters are intertwined, meaning that the breaking of one cluster implies that all other clusters also break. The possibility of observing the solution shown in either Fig. $7 b$ or e depends on their corresponding stability, which can change with $\sigma_{2}$. If more than one cluster is stable for a given value of $\sigma_{2}$, the observed solution depends on the initial condition.

Figure $7 \mathrm{~g}$ shows the MLEs $\Lambda_{q}$ corresponding to cluster $C_{q}(q=$ $1, \ldots, Q$ ) vs. $\sigma_{2}$ (again, all the curves overlap because the clusters are intertwined and the same MLE determines the stability of all clusters for each value of $\sigma_{2}$ ): it is evident that all these clusters are stable for $\sigma_{2}$ exceeding a threshold $\hat{\sigma} \approx 2.2$.

Figure $7 \mathrm{~h}$, i (obtained for $\sigma_{2}=2.3$ but with different initial conditions) shows time plots of the first component of the $q$ th cluster state $\boldsymbol{s}_{q}(t)$, for layer I (upper traces) and layer II (lower traces). Traces are colored as the corresponding clusters in Fig. $7 \mathrm{~b}, \mathrm{e}$, respectively. In Fig. $7 \mathrm{i}$ we see that almost each cluster corresponds to a different time trace, with the exception of the pairs $\left\{C_{1}, C_{3}\right\}$ and $\left\{C_{7}, C_{9}\right\}$, which are synchronized: this means that, in the original network, nodes 1,3 , and 9 are in a cluster and $11,13,19$ in a different one. By contrast, in Fig. $7 \mathrm{~h}$, the traces correspond to the minimal balanced coloring. This indicates the presence of multistability for $\sigma_{2}=2.3$. We remark that also other stable patterns may emerge: for instance, by setting initial conditions such that the states of $\mathrm{C} 4$ and $\mathrm{C} 6$ are close, also $\mathrm{C} 4$ and C6 synchronize.

Now we consider the same network in Fig. 7a, but we remove all interlayer connections from the upper layer to the lower layer. 
a

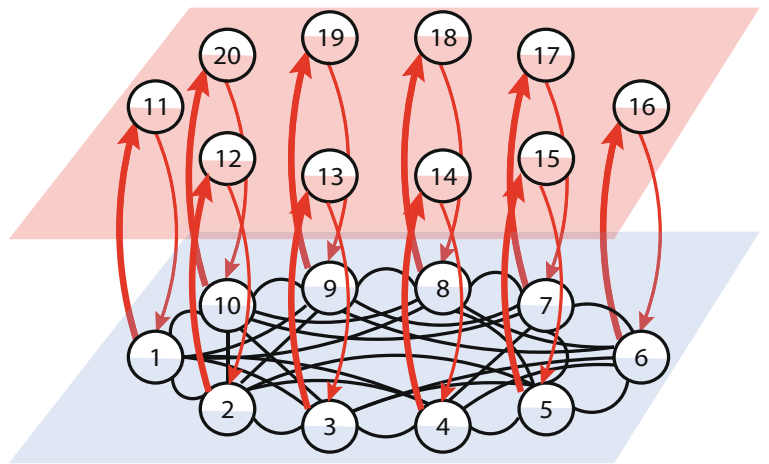

b
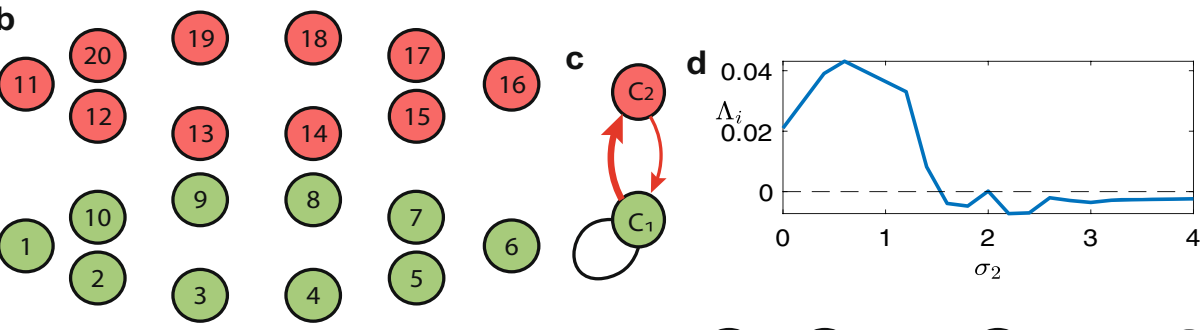

e

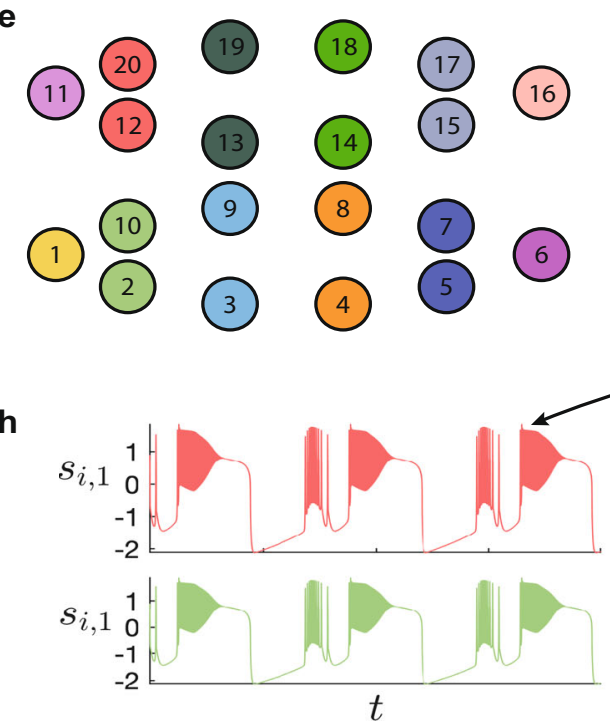

$\mathbf{f}$
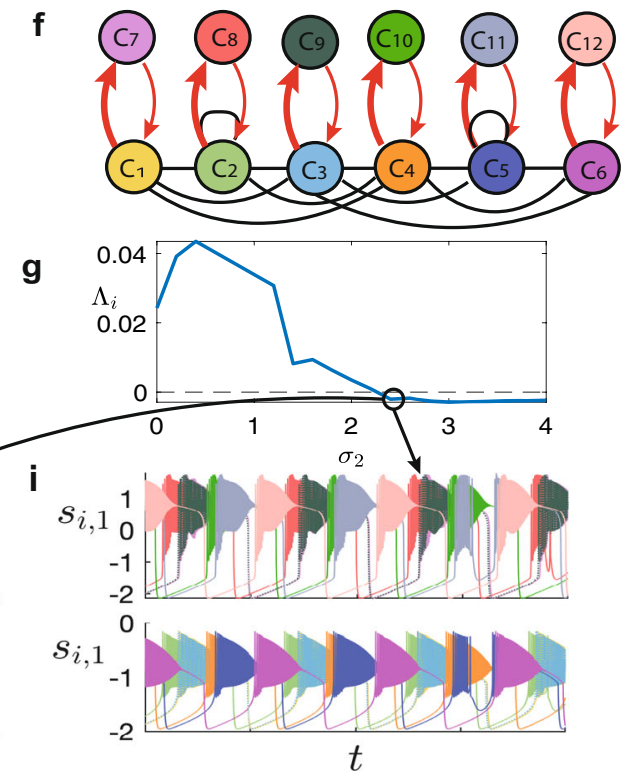

Fig. 7 Example adapted from ref. ${ }^{29}$. a Multilayer network with $N=20$ nodes, $L=2$ kinds of links, undirected intralayer and directed layer-to-layer connections. b Minimal balanced coloring/partition of the network, corresponding to $Q=2$ clusters $C_{1}$ (green nodes, from 1 to 10 ) and $C_{2}$ (red nodes, from 11 to 20). c Corresponding quotient network. d MLEs $\Lambda_{q}$ corresponding to cluster $C_{q}(q=1,2)$ vs. $\sigma_{2}$. e Non-minimal balanced coloring/partition, corresponding to $Q=12$ clusters, four of which are trivial (nodes $1,6,11,16$ ), while the other eight contain two nodes (2-10, 3-9, and so forth). f Corresponding quotient network. $\mathbf{g}$ MLEs $\Lambda_{q}$ corresponding to cluster $C_{q}(q=1, \ldots, Q)$ vs. $\sigma_{2}$. $\mathbf{h}, \mathbf{i}$ Time plots (first component of the synchronous cluster states $\boldsymbol{s}_{i}$ for $i=1, \ldots, Q$ ) evidencing the presence of multistability for $\sigma_{2}=2.3$ : $\mathbf{h}$ corresponds to the minimal balanced partition of $\mathbf{b}$ (two distinct traces), while $\mathbf{i}$ corresponds to the non-minimal balanced partition of $\mathbf{e}$ (twelve distinct traces).

In this case, the matrices $T$ and $B$ do not depend on $\sigma_{2}$. In both cases, we focus on the minimal balanced coloring only. By removing the connections from the upper to the lower layer, the $2 \times 2$ submatrix $B_{\perp}$ is block-upper triangular and the structure of the corresponding matrix $T$ evidences that cluster $C_{1}$ is one-waydependent on $C_{2}$ (see the Supplementary Note 3.4, for details). Conversely, if we remove the connections from the lower to the upper layer, i.e., the thick red connections from layer II to layer I, $B_{\perp}$ is again a unique $2 \times 2$ block-upper triangular submatrix and the structure of the corresponding matrix $T$ evidences that cluster $C_{2}$ is one-way-dependent on $C_{1}$.

Further details about the matrices obtained by applying our method are provided in Supplementary Data 2.

\section{Discussion}

There are two main contributions of this paper. We defined oneway-dependent clusters and provided a method to reduce the CS stability problem for directed networks of coupled nonlinear dynamical systems in an irreducible form.

The concept of one-way-dependent clusters is different from the previously reported concept of intertwined clusters, for which the desynchronization of a cluster $\mathrm{A}$ is subordinate to that of another cluster B and vice versa. As most real networks are directed, this concept has profound implications on our understanding of clustering in real-world settings. We illustrated this statement via two examples: a human network of violin players executing a musical piece, for which directed 
interactions may be either activated or deactivated by the musicians, and a multilayer neural network with directed layerto-layer connections.

Our work supersedes some of the limitations of previous research in the area of CS. Reference ${ }^{18}$ proposed a method that was effective in decoupling the CS stability problem in a number of irreducible lower-dimensional problems for arbitrary networks. However, the method only worked for orbital clusters and not also for equitable clusters. It is known ${ }^{53}$ that all orbital clusters are necessarily equitable but the converse is not necessarily true, so there exist equitable cluster partitions that are not orbital. There are notable examples of networks for which the minimal orbital cluster partition and the minimal equitable cluster partition do not coincide ${ }^{54}$. Reference ${ }^{27}$ focused on undirected and unweighted networks, including the case of equitable clusters, with the possibility of extending this theory to the case of directed and weighted networks. However, this method is unable to guarantee a decomposition of the stability problem in irreducible blocks. $\mathrm{In}^{24,43}$ an algebra-based approach is adopted to analyze the stability of synchronized clusters in undirected networks, as an alternative to the methods based on the theory of groups or groupoids ${ }^{16,18}$. In particular, the method presented in ref. ${ }^{24}$ is able to output irreducible blocks defined in a mathematically meaningful way, but does not apply to the case of directed networks. Explicit analytical conditions for the (local) stability of synchronized clusters in sparse and weighted networks of heterogeneous Kuramoto oscillators have been proposed in ref. ${ }^{55}$. To conclude, to the best of our knowledge, our method is the first one that permits to reduce the stability problem in a number of irreducible lower-dimensional subproblems for the important class of directed networks. As the model for the node dynamics is quite general ${ }^{56}$ and also the topology is now general (directed networks), the proposed method can be applied to a broad class of dynamical networks, overcoming the current limitations pointed out above. The proposed examples show the high flexibility of our approach.

Our method to build up the irreducible transformation matrix $T$ and the corresponding matrix $B$ is based in part on the methods for the stability analysis of CS solutions ${ }^{18,20-23,33}$ and in part on the methods for finding and analyzing ISS in networks of dynamical systems $19,34,38,40,41,57,58$. In reference to the latter class of methods, in particular, the correspondence between CS and the concepts of ISS and balanced partition was already established in refs. ${ }^{41,57}$. In ref. ${ }^{41}$ synchrony-breaking codimension-one bifurcations were analyzed using the ISS in weighted regular networks, namely networks in which the sum of the weights of the edges directed to the node is constant. However, these methods have been applied to classes of networks not as general as in this paper, even if ref. ${ }^{19}$ (which focuses on regular networks) provides some clues about generalization to non-regular and nonhomogeneous networks. We remark that the use of these tools for the stability analysis of the general class of networks (1) with optimal separation of the perturbation modes and for the associated construction of matrices $T$ and $B$ is new.

One of the main limitations of the proposed approach is its computational complexity, mainly due to the algorithm for finding the ISS ${ }^{19,34,59}$. As stated above, in the case of undirected networks, some methods (based either on the IRR of the symmetry group or on simultaneous block diagonalization of matrices) have been successfully applied to reduce the computational complexity of computing the transformation matrix $T$. Reducing the computational burden in the case of directed networks remains an open challenge.

Our method provides a key for characterizing model-based networks of dynamical systems and for analyzing CS (for networks of persistently oscillating elements, as in the case studies) or consensus ${ }^{60}$ (if the nodes of the network converge to an equilibrium point instead of a periodic solution) in directed networks. Among the many possible applications cited in the introduction, we believe that one of the most promising is in the area of neuroscience. The field of network neuroscience ${ }^{61}$ is one of the emerging frontiers in the investigation of complex brain functions. Principles and mechanisms underlying some of these functions may be related to the emergence of CS in directed networks.

\section{Methods}

Invariant synchrony subspaces (ISS). A subspace $U$ of $\mathbb{R}^{n}$ is invariant under a matrix $A \in \mathbb{R}^{n \times n}$ if $A U=\{A u: u \in U\}$ is a subset of $U$.

The ISS have been found following the theory developed in refs. $19,38,40,41,58$ and using the algorithm proposed in ref. ${ }^{34}$.

Proof of Eq. (6). We first recall that the CS manifold is $A^{k}$ invariant ${ }^{20}$ : this means that the image through $A^{k}$ of any vector that lies in the cluster-synchronous subspace (any linear combination of the first $Q$ rows of the matrix $T$ ) lies in the space spanned by the first $Q$ rows of the matrix $T$. Since

$$
B^{k}=T A^{k} T^{-1}
$$

and $T$ is orthonormal, we obtain

$$
A^{k} T^{T}=T^{T} B^{k}=\left(\left(B^{k}\right)^{T} T\right)^{T} .
$$

Recalling that the last $N-Q$ rows of $T$ are orthogonal to the first $Q,\left(B^{k}\right)^{T}$ must have 0 entries in all the last $N-Q$ columns of the first $Q$ rows. This means that the block $T_{\perp} A^{k} T_{\|}^{T}$ in Eq. (5) is null and therefore $B^{k}$ is block-upper triangular.

Models of example 2. Following ref. ${ }^{29}$, the equations governing the dynamics of layer I are

$$
\begin{gathered}
\dot{x}_{\frac{N}{2}+i}=a x_{\frac{N}{2}+i}^{2}-x_{\frac{N}{2}+i}^{3}-y_{\frac{N}{2}+i}-z_{\frac{N}{2}+i}+\sigma_{2}\left(d-x_{\frac{N}{2}+i}\right) h^{2}\left(x_{i}\right) \\
\dot{y}_{\frac{N}{2}+i}=(a+\alpha) x_{\frac{N}{2}+i}^{2}-y_{\frac{N}{2}+i} \\
\dot{z}_{\frac{N}{2}+i}=c\left(b x_{\frac{N}{2}+i}-z_{\frac{N}{2}+i}+e\right)
\end{gathered},
$$

where $a=2.8, \alpha=1.6, b=9, c=0.001, d=2, e=5$,

$$
h^{2}(x)=\frac{1}{1+e^{-\lambda(x-\Theta)}},
$$

$\lambda=10, \Theta=-0.25$

Similarly, the equations governing the dynamics of layer II are the following:

$$
\begin{gathered}
\dot{x}_{i}=a x_{i}^{2}-x_{i}^{3}-y_{i}-z_{i}+\sigma_{1} \sum_{j \in N_{s}} h^{1}\left(x_{i}, x_{j}\right)+\sigma_{2}\left(d-x_{i}\right) h^{2}\left(x_{\frac{N}{2}+i}\right) \\
\dot{y}_{i}=(a+\alpha) x_{i}^{2}-y_{i} \\
\dot{z}_{i}=c\left(b x_{i}-z_{i}+e\right)
\end{gathered},
$$

where $h^{1}\left(x_{i}, x_{j}\right)=x_{j}-x_{i}$ and $N_{S}$ is the neighborhood set of the $P$ neurons adjacent to neuron $i$. The other parameters are set to the same values as above, with the exception of $\alpha=1.7$, in order to have different neuron models in the two layers.

\section{Data availability}

All data generated and analyzed in this study are included in this published article (and its Supplementary Information files).

\section{Code availability}

The source code for the numerical simulations presented in the paper will be made available upon request.

Received: 29 January 2021; Accepted: 16 June 2021; Published online: 01 July 2021

\section{References}

1. Pikovsky, A., Rosenblum, M. \& Kurths, J. Synchronization: A Universal Concept in Nonlinear Sciences, Vol. 12 (Cambridge University Press, 2003).

2. Shlens, J., Rieke, F. \& Chichilnisky, E. Synchronized firing in the retina. Curr. Opin. Neurobiol. 18, 396-402 (2008).

3. Kiss, I. Z., Zhai, Y. \& Hudson, J. L. Emerging coherence in a population of chemical oscillators. Science 296, 1676-1678 (2002). 
4. Stone, L., Olinky, R., Blasius, B., Huppert, A. \& Cazelles, B. Complex synchronization phenomena in ecological systems. in AIP Conference Proceedings, Vol. 622, 476-488 (American Institute of Physics, 2002).

5. Grainger, J. \& Stevenson, W. Power System Analysis (McGraw-Hill Education, 1994).

6. Guevara Erra, R., Perez Velazquez, J. L. \& Rosenblum, M. Neural synchronization from the perspective of non-linear dynamics. Front. Comput. Neurosci. 11, 98 (2017).

7. Grillner, S. Biological pattern generation: the cellular and computational logic of networks in motion. Neuron 52, 751-766 (2006).

8. Ijspeert, A. J. Central pattern generators for locomotion control in animals and robots: a review. Neural Netw. 21, 642-653 (2008).

9. Goulding, M. Circuits controlling vertebrate locomotion: moving in a new direction. Nat. Rev. Neurosci. 10, 507-518 (2009).

10. Orosz, G., Wilson, R. E., Szalai, R. \& Stépán, G. Exciting traffic jams: Nonlinear phenomena behind traffic jam formation on highways. Phys. Rev. E 80, 046205 (2009).

11. Kaneko, K. Relevance of dynamic clustering to biological networks. Phys. D: Nonlinear Phenom. 75, 55-73 (1994).

12. Buono, P.-L. \& Collera, J. A. Symmetry-breaking bifurcations in rings of delay-coupled semiconductor lasers. SIAM J. Appl. Dyn. Syst. 14, 1868-1898 (2015).

13. Buono, P.-L. et al. Symmetry-breaking bifurcations and patterns of oscillations in rings of crystal oscillators. SIAM J. Appl. Dyn. Syst. 17, 1310-1352 (2018).

14. Kiss, A. K., Avedisov, S. S., Bachrathy, D. \& Orosz, G. On the global dynamics of connected vehicle systems. Nonlinear Dyn. 96, 1865-1877 (2019).

15. Golubitsky, M., Stewart, I. \& Török, A. Patterns of synchrony in coupled cell networks with multiple arrows. SIAM J. Appl. Dyn. Syst. 4, 78-100 (2005).

16. Golubitsky, M. \& Stewart, I. Nonlinear dynamics of networks: the groupoid formalism. Bull. Am. Math. Soc. 43, 305-364 (2006).

17. Golubitsky, M., Stewart, I. \& Schaeffer, D. G. Singularities and Groups in Bifurcation Theory, Vol. 2 (Springer Science \& Business Media, 2012).

18. Pecora, L. M., Sorrentino, F., Hagerstrom, A. M., Murphy, T. E. \& Roy, R. Cluster synchronization and isolated desynchronization in complex networks with symmetries. Nat. Commun. 5, 4079 (2014).

19. Aguiar, M. A. \& Dias, A. P. S. The lattice of synchrony subspaces of a coupled cell network: Characterization and computation algorithm. J. Nonlinear Sci. 24, 949-996 (2014)

20. Schaub, M. T. et al. Graph partitions and cluster synchronization in networks of oscillators. Chaos: Interdiscip. J. Nonlinear Sci. 26, 094821 (2016).

21. Sorrentino, F., Pecora, L. M., Hagerstrom, A. M., Murphy, T. E. \& Roy, R. Complete characterization of the stability of cluster synchronization in complex dynamical networks. Sci. Adv. 2, e1501737 (2016).

22. Siddique, A. B., Pecora, L., Hart, J. D. \& Sorrentino, F. Symmetry-and inputcluster synchronization in networks. Phys. Rev. E 97, 042217 (2018).

23. Della Rossa, F. et al. Symmetries and cluster synchronization in multilayer networks. Nat. Commun. 11, 1-17 (2020).

24. Zhang, Y. \& Motter, A. E. Symmetry-independent stability analysis of synchronization patterns. SIAM Rev. 62, 817-836 (2020).

25. Kirst, C., Timme, M. \& Battaglia, D. Dynamic information routing in complex networks. Nat. Commun. 7, 1-9 (2016)

26. Hens, C., Harush, U., Haber, S., Cohen, R. \& Barzel, B. Spatiotemporal signal propagation in complex networks. Nat. Phys. 15, 403-412 (2019)

27. Cho, Y. S., Nishikawa, T. \& Motter, A. E. Stable chimeras and independently synchronizable clusters. Phys. Rev. Lett. 119, 084101 (2017).

28. Shahal, S. et al. Synchronization of complex human networks. Nat. Commun. 11, 1-10 (2020).

29. Majhi, S., Perc, M. \& Ghosh, D. Chimera states in a multilayer network of coupled and uncoupled neurons. Chaos: Interdiscip. J. Nonlinear Sci. 27, 073109 (2017).

30. Ruzzene, G. et al. Remote pacemaker control of chimera states in multilayer networks of neurons. Phys. Rev. E 102, 052216 (2020).

31. Boccaletti, S. et al. The structure and dynamics of multilayer networks. Phys. Rep. 544, 1-122 (2014).

32. Kivelä, M. et al. Multilayer networks. J. Complex Netw. 2, 203-271 (2014).

33. Lodi, M., Della Rossa, F., Sorrentino, F. \& Storace, M. Analyzing synchronized clusters in neuron networks. Sci. Rep. 10, 16336 (2020).

34. Neuberger, J. M., Sieben, N. \& Swift, J. W. Invariant synchrony subspaces of sets of matrices. SIAM J. Appl. Dynamical Syst. 19, 964-993 (2020).

35. McKay, B. D. Practical Graph Isomorphism. Technical Report (1981).

36. Belykh, I. \& Hasler, M. Mesoscale and clusters of synchrony in networks of bursting neurons. Chaos: Interdiscip. J. Nonlinear Sci. 21, 016106 (2011).

37. Lodi, M., Della Rossa, F., Sorrentino, F. \& Storace, M. An algorithm for finding equitable clusters in multi-layer networks. In Proc. IEEE International Symposium on Circuits and Systems (ISCAS), 1-5 (2020).

38. Stewart, I., Golubitsky, M. \& Pivato, M. Symmetry groupoids and patterns of synchrony in coupled cell networks. SIAM J. Appl. Dyn. Syst. 2, 609-646 (2003).
39. Steur, E., Unal, H. U., van Leeuwen, C. \& Michiels, W. Characterization and computation of partial synchronization manifolds for diffusive delay-coupled systems. SIAM J. Appl. Dyn. Syst. 15, 1874-1915 (2016).

40. Aguiar, M. A., Dias, A. P. S. \& Ferreira, F. Patterns of synchrony for feedforward and auto-regulation feed-forward neural networks. Chaos: Interdiscip. J. Nonlinear Sci. 27, 013103 (2017).

41. Aguiar, M. A. \& Dias, A. P. S. Synchronization and equitable partitions in weighted networks. Chaos: Interdiscip. J. Nonlinear Sci. 28, 073105 (2018).

42. Pecora, L. M. \& Carroll, T. L. Master stability functions for synchronized coupled systems. Phys. Rev. Lett. 80, 2109 (1998).

43. Irving, D. \& Sorrentino, F. Synchronization of dynamical hypernetworks: dimensionality reduction through simultaneous block-diagonalization of matrices. Phys. Rev. E 86, 056102 (2012).

44. Tinkham, M. Group Theory and Quantum Mechanics (Courier Corporation, 2003).

45. Cuthill, E. \& McKee, J. Reducing the bandwidth of sparse symmetric matrices. In Proc. 24th Nat. Conf. ACM, 157-172 (1969).

46. Kuramoto, Y. \& Battogtokh, D. Coexistence of coherence and incoherence in nonlocally coupled phase oscillators. Nonlinear Phenom. Complex Syst. 5, 380-385 (2002).

47. Abrams, D. M. \& Strogatz, S. H. Chimera states for coupled oscillators. Phys. Rev. Lett. 93, 174102 (2004).

48. Majhi, S., Bera, B. K., Ghosh, D. \& Perc, M. Chimera states in neuronal networks: a review. Phys. Life Rev. 28, 100-121 (2019).

49. Wang, Z. \& Liu, Z. A brief review of chimera state in empirical brain networks. Front. Physiol. 11, 724 (2020).

50. Ayala, G., Dichter, M., Gumnit, R., Matsumoto, H. \& Spencer, W. Genesis of epileptic interictal spikes. New knowledge of cortical feedback systems suggests a neurophysiological explanation of brief paroxysms. Brain Res. 52, 1-17 (1973).

51. Mukhametov, L., Supin, A. Y. \& Polyakova, I. Interhemispheric asymmetry of the electroencephalographic sleep patterns in dolphins. Brain Res. 134, 581-584 (1977)

52. Rattenborg, N. C., Amlaner, C. \& Lima, S. Behavioral, neurophysiological and evolutionary perspectives on unihemispheric sleep. Neurosci. Biobehav. Rev. 24, 817-842 (2000).

53. Bari, R. A. \& Harary, F. Graphs and Combinatorics: Proceedings of the Capital Conference on Graph Theory and Combinatorics at the George Washington University, June 18-22, 1973, Vol. 406 (Springer, 2006).

54. Kudose, S. Equitable partitions and orbit partitions. (2009). available at: http://www.math.uchicago.edu/ may/VIGRE/VIGRE2009/REUPapers/ Kudose.pdf.

55. Menara, T., Baggio, G., Bassett, D. S. \& Pasqualetti, F. Stability conditions for cluster synchronization in networks of heterogeneous Kuramoto oscillators. IEEE Trans. Control Netw. Syst. 7, 302-314 (2020).

56. Barzel, B. \& Barabási, A.-L. Universality in network dynamics. Nat. Phys. 9 673-681 (2013).

57. Aguiar, M. A., Dias, A. P. S., Golubitsky, M. \& Maria da Conceição, A. L. Bifurcations from regular quotient networks: a first insight. Phys. D: Nonlinear Phenom. 238, 137-155 (2009).

58. Aguiar, M., Ashwin, P., Dias, A. \& Field, M. Dynamics of coupled cell networks: synchrony, heteroclinic cycles and inflation. J. Nonlinear Sci. 21 271-323 (2011).

59. Kamei, H. \& Cock, P. J. Computation of balanced equivalence relations and their lattice for a coupled cell network. SIAM J. Appl. Dyn. Syst. 12, 352-382 (2013).

60. Sorrentino, F., Pecora, L. M. \& Trajkovic, L. Group consensus in multilayer networks. IEEE Trans. Netw. Sci. Eng. 7, 2016-2026 (2020).

61. Bassett, D. S. \& Sporns, O. Network neuroscience. Nat. Neurosci. 20, 353-364 (2017).

\section{Acknowledgements}

The authors would like to express their sincere appreciation to Fabio Della Rossa for many useful inputs and valuable comments. They also thank Martin Golubitsky, Mauro Parodi, and Louis Pecora for reading the first version of this manuscript and providing constructive comments. F.S. acknowledges support from NIH grant 1R21EB028489.

\section{Author contributions}

F.S. and M.S. designed and supervised the research; M.L. performed the research; M.L. F.S., and M.S. analyzed the data and interpreted the results; M.S. wrote and revised the manuscript; M.L. and F.S. contributed to writing and revised the manuscript.

\section{Competing interests}

The authors declare no competing interests. 


\section{Additional information}

Supplementary information The online version contains supplementary material available at https://doi.org/10.1038/s41467-021-24363-7.

Correspondence and requests for materials should be addressed to M.S.

Peer review information Nature Communications thanks Moti Fridman and the other anonymous reviewer(s) for their contribution to the peer review of this work.

Reprints and permission information is available at http://www.nature.com/reprints

Publisher's note Springer Nature remains neutral with regard to jurisdictional claims in published maps and institutional affiliations. (c) (P) Open Access This article is licensed under a Creative Commons Attribution 4.0 International License, which permits use, sharing, adaptation, distribution and reproduction in any medium or format, as long as you give appropriate credit to the original author(s) and the source, provide a link to the Creative Commons license, and indicate if changes were made. The images or other third party material in this article are included in the article's Creative Commons license, unless indicated otherwise in a credit line to the material. If material is not included in the article's Creative Commons license and your intended use is not permitted by statutory regulation or exceeds the permitted use, you will need to obtain permission directly from the copyright holder. To view a copy of this license, visit http://creativecommons.org/licenses/by/4.0/.

(C) The Author(s) 2021 